\title{
Interactive comment on "Evidence for elevation-dependent warming from the Chinese Tianshan Mountains” by Lu Gao et al.
}

\section{Anonymous Referee \#3}

Received and published: 5 November 2020

Gao and co-authors present an analysis of decadal air temperature trends against elevation to explore the case for elevation dependent warming (EDW) in the Chinese Tianshan Mountains (CTM). The authors explore this across a large domain using a recent $1 \mathrm{~km}$ resolution product derived based upon ERA-Interim reanalysis and station data up to $3000 \mathrm{~m}$ a.s.l. They find that for given months and sub-domains of the CTM, EDW is evident, though is complex and not consistent or clear for all domains or seasons. The manuscript is well written in parts and explores a very interesting and relevant topic within the cryosphere. While the work has particular value to be published in the journal, I believe much more needs to be done to explain the data sources and their limitations, to convince the reader of the validity of CTMD product and therefore the uncertainty and limitations of their results as well as providing more 
justification and better presentation of the key findings.

General Comments I think the manuscript has promise and could be substantially improved based upon some key things.

1) The authors give general reference to their ESSD paper for details about the CMTD product, but a much stronger section of the data and methods need to be presented for this manuscript in order to summarise the key details about how the CMTD was derived, for what time scale it is processed and what the major assumptions or limitations are that might affect the analysis of EDW. It's apparent to me that the authors are already considering these limitations etc, based upon their responses to other reviewers on the open-discussions. To the reader of this manuscript, there is not enough information presented to judge the quality of the CTMD and assess the validity of the results that are based upon it.

2) I have the same issue as 1), but also for the CMA05 product. I am left questioning the comparability of the two for the tabular information presented (the first criterion of EDW that is the regionally amplified warming). For the CMA05, all pixels are averaged to produce a temperature/warming trend for all elevations across the entirety of China? Is this dataset also derived from ERA-I? Does it include the CTM as well, or all the rest of China except the study domain? If it is all of China, this then also includes other mountain regions of the country? In general, I like the succinct and to-the-point paper, but there are a lot of important pieces of information that are missing and without them, the reader cannot gain a good appreciation of the scientific rigour and value of the authors work. Being clearer about some of those elements will greatly aid the scientific conclusions.

3) In some places, a justification for showing some months and not others are needed. Figures for Tmin, Tmax and Tmean all show different months, for example. Is this purely just to show the months with the strongest trends? Some work needs to go into the figures as well. I see that that has begun already based upon comments from

Printer-friendly version

Discussion paper 
reviewer\#2. In each figure, the authors show different scales (y-axis limits are different in Figures 2-4 and colour scales are different in each subplot for Figures 5-7), and it becomes hard for the reader to easily compare and understand them, and take away the key message(s). See specific comments on the figures below.

4) The manuscript presents a rather general discussion with little further exploration of possible mechanisms. There is a repetition of general comments regarding, for example, the albedo's role on the surface energy balance, but this never links with why we may see EDW in certain months or why the strongest warming may occur only for Tmin in January/December and why Tmax trends or regional (east-west) temperature trends (e.g. Figure 5) might occur. A reference of Deng et al. 2019 is given, for example, but it is not elaborated upon much. Can this or other datasets or analyses regarding snow cover/albedo from MODIS tell us more about why EDW might be occurring for certain seasons/mountains/zones? I don't suggest that the authors do a full analysis of snow cover, but some additional and more in-depth discussion points are definitely required.

5) Finally, throughout the manuscript, the terminology of EDW and trends/gradients shifts somewhat and consistency is required throughout (following a clear initial definition). Moreover, the use of the word 'significantly' comes up a lot to refer to differences in trends across space (for the maps) and time (for seasons / months). Unless these differences are tested for significance and values reported, care should be taken for the wording and adjusted appropriately.

Specific Comments

Abstract L26 -What are EDW 'Features'? I would consider rewording this. L26-27 Please add here the time period over which CMTD was derived and analysed (19792016?) L28 - Statistically significant elevation dependence? Add that if so. L34 While I do not disagree that this is a likely contributor to glacier melt in the CTM, the authors do not explicitly 'explain' this link, especially as the EDW trends are not so clear for all summer months. It's possible that stronger trends in warming at high elevations

Printer-friendly version

Discussion paper 
in April could have a key influence on some more precipitation falling as rain, but again, the authors cannot (based upon the presented work) state this. I would rephrase this to something like "This new evidence could partly explain the accelerated melting of glaciers in the CTM, though the mechanisms remain to be explored" or similar.

Introduction

L36 - two 'criteria

L50 - Current 'evidence'

L54 - Please elaborate here and add some reasoning of seasonal significance from those studies.

L58 - What is global mountain detection? Do the authors refer to detection of trends or 'observations' in general for mountain regions? Please clarify and reword.

L58-74 This paragraph reads rather disjointed without a clear flow or argument. Because it recounts several other instances of studies exploring EDW, the overview might be more valuable to the reader in a tabular format? I would suggest to restructure this paragraph and improve the flow of the writing.

L72-73 - Please clarify what satellite data the authors refer to and how that shows EDW/climate warming at specified elevations. How does this point fit into the context of the manuscript discussion and/or the strengths/limitations of the presented dataset?

L81 - Do the authors refer to 56 gridded points of a given product presented by You et al.? Please clarify and rewrite.

L87 - To me the "largest independent latitudinal mountain system" is not clear. Can the authors clarify its meaning or remove it?

Printer-friendly version

Data and Methods

L109 - CTMD is briefly defined at the end of the introduction, but should be described in 
sufficient detailed before introducing other datasets to compare to it. See my general comment about elaborating on the CTMD product, especially on its derivation and potential limitations for exploring EDW in this manuscript.

L111-Taking all elevations of CMA05? It is not clear how comparable these products are (see general comment). For the CTMD product, the definition of mountain domain is all of the CTMD pixels (including low elevations)? I am left questioning whether the comparison of the CTMD and CMA05 trends are valid and how the values for Table 1 were derived for each of them. More information is required here.

L112 - Can the authors define what is a small large scale error? Small biases over large domains?

L113 - systematic?

L116-118 - It would be valuable to recount that winter lapse rates were not well estimated by CTMD compared to the station data as shown by Fig. 4 of Gao et al., 2018. Some mention here (or in the discussion) needs to explore the potential impact that this might have on your results. If, for example, your temperatures at the highest elevations were estimated by the station lapse rates, would they be largely different from what the CTMD gives you? Could this strongly affect the EDW trends for the highest elevations in January/December? I don't expect that the authors should use the low-elevation stations to derive the high elevation temperatures for their analyses, but some discussion on the limitations of CTMD for the current analyses are required somewhere in the manuscript.

L126 - reword to 'six-hourly timestep'

L136-138 - fine, but maybe neaten, use of table?

Printer-friendly version

L139 - statistical significance of the linear regression? What p-value defines your statistical significance when you use the term significant in the abstract?

Discussion paper

L141- averaged is mean or median? (cf boxplots with median red line plotted) 


\section{Results}

L150 - This needs clarification. Do the authors refer to the elevation gradient of decadal temperature trends or the gradient (slope) of the regression line that quantifies the trend in each elevation band? If referring to the latter, please use the word trend (or similar) instead to not confuse with temperature gradient/lapse rate.

L174 - Why those months only? How are they 'representative'? Representative of what? I don't see a clear segregation of season, January and December both have negative trends for the whole domain (converse to the CMA05), April is not as large an increase as March... More justification is needed. Are the authors simply showing all of the results which have more warming somewhere?

L176 - Is your average a Mean? Median? Note that median is displayed for boxplots.

L185 - Figure 3 now investigates March, April, August and September. Why are the same months not compared and what is the justification this time?

L193 - Months of interest for Tmean are again different.

L203 - Statistically significantly different? If so, by what test and what significance? Same comment throughout the paragraph, please clarify the significance or reword it.

L207 - are warmer on average, the figure rather shows a higher rate of warming. Check sentence.

Possible hypotheses and mechanisms

I feel that this section should be under the general header of 'discussion'. Please see general comments on this section. I believe that much more is needed for this section. It is very general and I don't go away feeling that I learned anything new.

L255 - Also the snow cover and snow albedo here affect this... This is mentioned in the next paragraph and the information is essentially repeated with no additional information gain. 
L264-265 - Sure, this could be a mechanism, but has there been any other studies demonstrating snow cover changes and albedo changes in the CTM? I note that the Deng paper is cited but not investigated further. Because the CTMD product is generated through station observations at lower elevations, would this not bias representation of high elevation changes? Of course, I appreciate that there are no available data at those higher elevations, but this needs to be mentioned and limitations of the dataset/study need to be linked with a more in depth interpretation of the most noteworthy results.

L273 - Could be? Are these model simulations of idealised conditions or did authors find this specifically for that zone? Reword to 'estimated glacier mass loss...'

\section{L275 - 'In summary'}

\section{Discussion and Conclusions}

In my opinion, this section needs splitting into; 1) a greater discussion with section 4 (see general comment and above) and, 2) a clear and concise, separate conclusions section.

L284 - 'DO' not (in the case of CTM) clearly reflect EDW. Not cannot.

L285-286 - This belongs to the previous section. The authors should elaborate whether earlier spring snow melt is significant (and quantify significance) or at least demonstrate if past work suggests that warming at those higher elevations is more likely. Comparing some general estimates of snow line elevation or from previous findings to those same elevation bands would be of value, though I'm sceptical if the CTMD product will reflect that change.

L288 - Replace gradients with trends unless referring specifically to the difference across the elevation bands (Figures 2-4). In general, the terminology needs clarification.

L297 - I think that this is a crucial point. Above $5000 \mathrm{~m}$, there are always positive 
changes... but for lower elevations in those winter months, there are largely negative trends for minimum and some mean temperatures (Figures 2 and 4). I would like to see more discussion as to why we might expect to have a general cooling (negative) trend for the winter minimum below $3000 \mathrm{~m}$. The lack of discussion regarding the mechanisms is a major drawback to the current manuscript version.

L297-298 - Or could be warming as a result of snow cover depletion (feedback)?

L297-302 - This reads like a results section again.

\section{Figures}

-My general issue with the figures is the lack of standardisation (i.e. different colour and $y$-axis scales) and the ever changing months presented. It leaves the reader with no strong idea as to the key findings.

-I recommend maintaining the same y-axis limits to all sub-plots in Figures 2-4, labelling the months on the plots for easier interpretation.

-For Figures 5-7, please adjust the colour scale from left (blue - negative) to right (red - positive) following the reviewer\#2 comments and also set the same total scale limit for each plot (i.e. $-1.5-+1.5^{\circ} \mathrm{C} 10 \mathrm{a}-1$ ) with $0^{\circ} \mathrm{C}$ trend always being the same colour (pale yellow or white). Do the authors also report trends that are not statistically significant? If so, I would also represent these as white or blank pixels if possible. This will aid the reader's ability to interpret and compare the magnitudes of trends between sub-plots/figures as well as areas that aren't statistically significant trends.

- I would suggest adding some other figure(s) that shows the interannual variability of Tmin/Tmax/Tmean for some of the highest elevation pixels so we can better interpret how the suspected EDW warming for March/April/(or month of most interest) looks compared to some lower elevations, or compared to the 'background' change of 'non-mountain' regions shown from the CMA05, if the CMA05 and CTMD are indeed comparable (see general comment). These are the two criteria for EDW and need to

Printer-friendly version

Discussion paper 
be more convincingly demonstrated and discussed.

Interactive comment on The Cryosphere Discuss., https://doi.org/10.5194/tc-2020-188, 2020.

Interactive

comment 